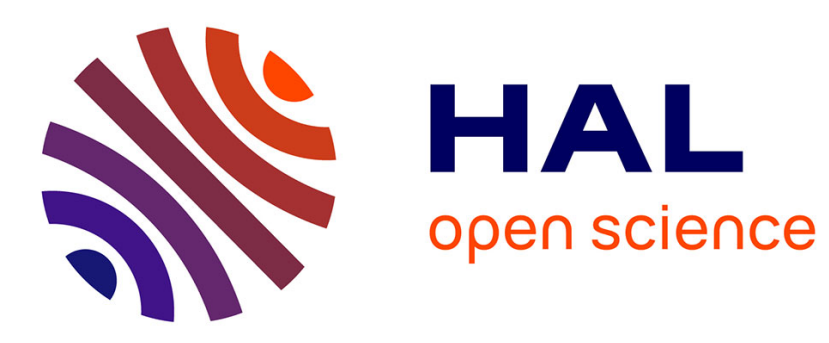

\title{
On fixed-time parameter estimation under interval excitation
}

Denis Efimov, Stanislav Aranovskiy, Alexey Bobtsov, Tarek Raïssi

\section{To cite this version:}

Denis Efimov, Stanislav Aranovskiy, Alexey Bobtsov, Tarek Raïssi. On fixed-time parameter estimation under interval excitation. ECC 2020 - 18th European Control Conference, May 2020, SaintPetersburg, Russia. hal-02634489

\section{HAL Id: hal-02634489 \\ https://hal.inria.fr/hal-02634489}

Submitted on 27 May 2020

HAL is a multi-disciplinary open access archive for the deposit and dissemination of scientific research documents, whether they are published or not. The documents may come from teaching and research institutions in France or abroad, or from public or private research centers.
L'archive ouverte pluridisciplinaire HAL, est destinée au dépôt et à la diffusion de documents scientifiques de niveau recherche, publiés ou non, émanant des établissements d'enseignement et de recherche français ou étrangers, des laboratoires publics ou privés. 


\title{
On fixed-time parameter estimation under interval excitation
}

\author{
D. Efimov, S. Aranovskiy, A.A. Bobtsov, T. Raïssi
}

\begin{abstract}
The problem of estimation in the linear regression model is studied under the hypothesis that the regressor may be excited on a limited initial interval of time only. Then the estimation solution is based on the framework of finite-time or fixed-time converging dynamical systems. Two estimation algorithms are proposed. The robustness is analyzed using the notion of short-time input-to-state stability property with the use of a Lyapunov function. The performance of the estimators is demonstrated in numerical experiments.
\end{abstract}

\section{INTRODUCTION}

One of the basic and the most popular problems in the theory of identification and estimation is the parameter estimation in a linear regression model:

$$
y(t)=\omega^{\top}(t) \theta+w(t), t \in \mathbb{R},
$$

where $\theta \in \mathbb{R}^{n}$ is the vector of unknown constant parameters that is necessary to find, $\omega: \mathbb{R} \rightarrow \mathbb{R}^{n}$ is the regressor function (usually assumed to be bounded and known), $y(t) \in \mathbb{R}$ is the signal available for measurements with a measurement noise $w: \mathbb{R} \rightarrow \mathbb{R}$ (here $\mathbb{R}$ denotes the set of real numbers). There are plenty of methods to solve this problem that need a complete statistics on the process (in other words these tools are mainly oriented on estimation offline): the linear least squares, the maximum-likelihood estimation, the Bayesian linear regression, the principal component regression [1], [2], to mention a few. In the theory of adaptive control and identification there exist also many methods for adaptive and online estimation [3], [4], and applicability of many of them is based on the condition of persistence of excitation [5], [6]. Consequently, these approaches are also implicitly based on the asymptotic statistics. Recently, several concepts have been proposed to relax the requirement on the excitation [7], [8], [9], with improved estimation algorithms [10], [11], [12], [13], [14], which require only an interval estimation of the regressor $\omega(t)$.

Considering convergence on a finite interval, the amplitude of the initial error becomes of great importance, since if this deviation is not bounded, then it is complicated to ensure global convergence of the estimates to their ideal values in a limited time. A notion that overcomes this drawback has been proposed recently, and it is called fixed-time or predefined-time stability/convergence [15], [16], [17], [18]. In the present work we discuss several fixed-time convergent algorithms solving the parameter estimation problem in a linear regression model, which are independent in the initial guesses for the values of parameters and regressor excitation ${ }^{1}$, and

Denis Efimov is with Inria, Univ. Lille, CNRS, UMR 9189 - CRIStAL, F-59000 Lille, France.

Stanislav Aranovskiy is with CentaleSupélec-IETR, Avenue de la Boulaie, 35576 Cesson-Sévigné, France.

Alexey A. Bobtsov is with ITMO University, 49 av. Kronverkskiy, 197101 Saint Petersburg, Russia.

Tarek Raïssi is with Conservatoire National des Arts et Métiers (CNAM), Cedric 292, Rue St-Martin, 75141 Paris, France.

This work was supported by the Ministry of Science and Higher Education of Russian Federation, passport of goszadanie no. 2019-0898.

${ }^{1}$ In one of our previous studies [12] only a finite-time convergence has been guaranteed, therefore, the synthesized algorithms use an upper bound on the initial error. whose robustness against the measurement noise is assessed using the input-to-state stability (ISS) theory. Following [14], a notion of fixed-time input-to-state stability on a short interval of time is applied together with a Lyapunov function analysis. The considered approach is based on the dynamic regressor extension and mixing (DREM) method [19], [13], which allow the vector estimation problem to be decoupled on a series of scalar ones. The obtained solutions are illustrated by computer simulations.

\section{Notation}

- $\mathbb{R}_{+}=\{x \in \mathbb{R}: x \geq 0\}$, where $\mathbb{R}$ is the set of real numbers.

- $|x|$ denotes the absolute value for $x \in \mathbb{R}$ or a vector norm for $x \in \mathbb{R}^{n}$, and the corresponding induced matrix norm for a matrix $A \in \mathbb{R}^{n \times n}$ is denoted by $\|A\|$.

- For a Lebesgue measurable and essentially bounded function $x: \mathbb{R} \rightarrow \mathbb{R}^{n}$ denote $\|x\|_{\infty}=\operatorname{ess}_{\sup _{t \in \mathbb{R}}}|x(t)|$, and define by $\mathcal{L}_{\infty}\left(\mathbb{R}, \mathbb{R}^{n}\right)$ the set of all such functions with finite norms $\|\cdot\|_{\infty} ;$ if

$$
\int_{-\infty}^{+\infty}|x(t)|^{2} d t<+\infty
$$

then this class of functions is denoted by $\mathcal{L}_{2}\left(\mathbb{R}, \mathbb{R}^{n}\right)$.

- A continuous function $\alpha: \mathbb{R}_{+} \rightarrow \mathbb{R}_{+}$belongs to the class $\mathcal{K}$ if $\alpha(0)=0$ and the function is strictly increasing, a function $\alpha \in \mathcal{K}$ belongs to the class $\mathcal{K}_{\infty}$ if it is increasing to infinity. A function $\beta: \mathbb{R}_{+} \times \mathbb{R}_{+} \rightarrow \mathbb{R}_{+}$belongs to the class $\mathcal{K} \mathcal{L}$ if $\beta(\cdot, t) \in \mathcal{K}$ for each fixed $t \in \mathbb{R}_{+}$and $\beta(s, \cdot)$ is decreasing and $\lim _{t \rightarrow+\infty} \beta(s, t)=0$ for each fixed $s \in \mathbb{R}_{+}$; a function $\beta: \mathbb{R}_{+} \times \mathbb{R}_{+} \rightarrow \mathbb{R}_{+}$belongs to the class $\mathcal{G} \mathcal{K} \mathcal{L}$ if $\beta(s, 0) \in \mathcal{K}$, $\beta(s, \cdot)$ is decreasing and for each $s \in \mathbb{R}_{+}$there is $T_{s} \in \mathbb{R}_{+}$ such that $\beta(s, t)=0$ for all $t \geq T_{s}$.

- The identity matrix of dimension $n \times n$ is denoted as $I_{n}$.

- Define $\mathbf{e}=\exp (1)$. Define the Lambert function Lambert : $\mathbb{R} \rightarrow \mathbb{R}$, also called the omega function or product logarithm, as the branches of the inverse relation of the function $f(z)=z \mathbf{e}^{z}$ for $z \in \mathbb{R}$ (in general it is introduced for $z \in \mathbb{C}$, but it has also two brances of solutions for $z \in \mathbb{R}$ ).

- Denote $\lceil s\rfloor^{\alpha}=|s|^{\alpha} \operatorname{sign}(s)$ for any $s \in \mathbb{R}$ and $\alpha \in \mathbb{R}_{+}$.

\section{Preliminaries}

Consider a time-dependent differential equation:

$$
d x(t) / d t=f(t, x(t), d(t)), t \geq t_{0}, t_{0} \in \mathbb{R},
$$

where $x(t) \in \mathbb{R}^{n}$ is the state vector, $d(t) \in \mathbb{R}^{m}$ is the vector of external inputs and $d \in \mathcal{L}_{\infty}\left(\mathbb{R}, \mathbb{R}^{m}\right) ; f: \mathbb{R}^{n+m+1} \rightarrow \mathbb{R}^{n}$ is a continuous function with respect to $x, d$ and piece-wise continuous with respect to $t, f(t, 0,0)=0$ for all $t \in \mathbb{R}$. A solution of the system (1) for an initial condition $x_{0} \in \mathbb{R}^{n}$ at time instant $t_{0} \in$ $\mathbb{R}$ and some $d \in \mathcal{L}_{\infty}\left(\mathbb{R}, \mathbb{R}^{m}\right)$ is denoted as $X\left(t, t_{0}, x_{0}, d\right)$, and we assume that $f$ ensures definiteness and uniqueness of solutions $X\left(t, t_{0}, x_{0}, d\right)$ in forward time at least on some finite time interval $\left[t_{0}, t_{0}+T\right)$, where $T>0$ may be dependent on the initial condition $x_{0}$, the input $d$ and the initial time $t_{0}$. 


\section{A. Stability definitions}

Let $\Omega, \Xi$ be open neighborhoods of the origin in $\mathbb{R}^{n}, 0 \in \Omega \subset \Xi$, then following [20], [12] introduce several stability notions:

Definition 1. At the steady state $x=0$ the system (1) with $d=0$ is said to be

(a) short-time stable with respect to $\left(\Omega, \Xi, T^{0}, T_{f}\right)$ if for any $x_{0} \in \Omega$ and $t_{0} \in\left[-T^{0}, T^{0}\right], X\left(t, t_{0}, x_{0}, 0\right) \in \Xi$ for all $t \in\left[t_{0}, t_{0}+\right.$ $\left.T_{f}\right]$

(b) short-finite-time stable with respect to $\left(\Omega, \Xi, T^{0}, T_{f}\right)$ if it is short-time stable with respect to $\left(\Omega, \Xi, T^{0}, T_{f}\right)$ and finite-time converging from $\Omega$ with the convergence time $T^{t_{0}, x_{0}} \leq t_{0}+T_{f}$ :

$$
\left|X\left(t, t_{0}, x_{0}, 0\right)\right|=0 \quad \forall t \geq T^{t_{0}, x_{0}},
$$

for all $x_{0} \in \Omega$ and $t_{0} \in\left[-T^{0}, T^{0}\right]$;

(c) globally short-finite-time stable for $T^{0}>0$ if for any bounded set $\Omega \subset \mathbb{R}^{n}$ containing the origin there exist a bounded set $\Xi \subset \mathbb{R}^{n}, \Omega \subset \Xi$ and $T_{f}>0$ such that the system is shortfinite-time stable with respect to $\left(\Omega, \Xi, T^{0}, T_{f}\right)$;

(d) short-fixed-time stable for $T^{0}>0$ and $T_{f}>0$, if for any bounded set $\Omega \subset \mathbb{R}^{n}$ containing the origin there exists a bounded set $\Xi \subset \mathbb{R}^{n}, \Omega \subset \Xi$ such that the system is short-finite-time stable with respect to $\left(\Omega, \Xi, T^{0}, T_{f}\right)$.

\section{B. Robust stability definitions}

Consider the following definition of robust stability for (1) with $d \neq 0$ [12], [14]:

Definition 2. The system (1) is said to be

(a) short-finite-time ISS with respect to $\left(\Omega, T^{0}, T_{f}, D\right)$ if there exist $\beta \in \mathcal{G K} \mathcal{L}$ and $\gamma \in \mathcal{K}$ such that for all $x_{0} \in \Omega$, all $d \in$ $\mathcal{L}_{\infty}\left(\mathbb{R}, \mathbb{R}^{m}\right)$ with $\|d\|_{\infty}<D$ and $t_{0} \in\left[-T^{0}, T^{0}\right]$ :

$\left|X\left(t, t_{0}, x_{0}, d\right)\right| \leq \beta\left(\left|x_{0}\right|, t-t_{0}\right)+\gamma\left(\|d\|_{\infty}\right) \quad \forall t \in\left[t_{0}, t_{0}+T_{f}\right]$ and $\beta\left(\left|x_{0}\right|, T_{f}\right)=0$;

(b) globally short-finite-time ISS for $T^{0}>0$ if there exist $\beta \in \mathcal{G K} \mathcal{L}$ and $\gamma \in \mathcal{K}$ such that for any bounded set $\Omega \subset \mathbb{R}^{n}$ containing the origin there is $T_{f}>0$ such that for all $x_{0} \in \Omega$, all $d \in \mathcal{L}_{\infty}\left(\mathbb{R}, \mathbb{R}^{m}\right)$ and $t_{0} \in\left[-T^{0}, T^{0}\right]:$

$\left|X\left(t, t_{0}, x_{0}, d\right)\right| \leq \beta\left(\left|x_{0}\right|, t-t_{0}\right)+\gamma\left(\|d\|_{\infty}\right) \quad \forall t \in\left[t_{0}, t_{0}+T_{f}\right]$

and $\beta\left(\left|x_{0}\right|, T_{f}\right)=0$ (the system is short-finite-time ISS with respect to $\left.\left(\Omega, T^{0}, T_{f},+\infty\right)\right)$;

(c) short-fixed-time ISS for $T^{0}>0$ and $T_{f}>0$, if there exist $\beta \in \mathcal{G K} \mathcal{L}$ and $\gamma \in \mathcal{K}$ such that for all $x_{0} \in \mathbb{R}^{n}$, all $d \in \mathcal{L}_{\infty}\left(\mathbb{R}, \mathbb{R}^{m}\right)$ and $t_{0} \in\left[-T^{0}, T^{0}\right]$ :

$\left|X\left(t, t_{0}, x_{0}, d\right)\right| \leq \beta\left(\left|x_{0}\right|, t-t_{0}\right)+\gamma\left(\|d\|_{\infty}\right) \quad \forall t \in\left[t_{0}, t_{0}+T_{f}\right]$ and $\beta\left(\left|x_{0}\right|, T_{f}\right)=0$ (for any bounded set $\Omega \subset \mathbb{R}^{n}$ containing the origin the system is short-finite-time ISS with respect to $\left.\left(\Omega, T^{0}, T_{f},+\infty\right)\right)$.

The difference of global short-finite-time or short-fixed-time ISS and a conventional (finite-time or fixed-time) ISS [21], [22] is that in the former case the stability property is considered on a finite interval of time $\left[t_{0}, t_{0}+T_{f}\right]$ only.

Theorem 1. [14] Let the constants $T^{0} \geq 0$ and $T_{f}>0$ be given. Let the system in (1) possess a Lyapunov function $V: \mathbb{R} \times \mathbb{R}^{n} \rightarrow \mathbb{R}_{+}$ such that for all $x \in \mathbb{R}^{n}, d \in \mathbb{R}^{m}$ and $t \in\left[-T^{0}, T^{0}+T_{f}\right]$ :

$$
\begin{gathered}
\alpha_{1}(|x|) \leq V(t, x) \leq \alpha_{2}(|x|) \quad \alpha_{1}, \alpha_{2} \in \mathcal{K}_{\infty} ; \\
\dot{V}(t, x) \leq-u(t)\left(V^{1-\eta}(t, x)+V^{1+\eta}(t, x)\right)+\kappa(|d|)
\end{gathered}
$$

for $\kappa \in \mathcal{K}, \eta \in(0,1)$ and a function $u: \mathbb{R} \rightarrow \mathbb{R}_{+}$satisfying

$$
\int_{t}^{t+\ell} u(s) d s \geq v>0, \ell>0
$$

for all $t \in\left[-T^{0}, T^{0}+T_{f}\right]$. Then the system (1) is short-fixed-time ISS for $T^{0}$ and $T_{f}$ with

$$
\begin{gathered}
\beta(s, t)=\alpha_{1}^{-1}\left(2 ^ { \eta ^ { - 1 } } \operatorname { m i n } \left\{\max \left\{0, \sqrt[\eta]{\alpha_{2}^{\eta}(s)-\eta \frac{v}{4 \ell}(t-\ell)}\right\},\right.\right. \\
\left.\left.\frac{1}{\sqrt[\eta]{\alpha_{2}^{-\eta}(s)+\eta \frac{v}{4 \ell}(t-\ell)}}\right\}\right), \\
\gamma(s)=\alpha_{1}^{-1}\left[2^{\eta^{-1}}\left(\ell \kappa(s)+\max \left\{\left(\frac{4 \ell}{v} \kappa(s)\right)^{\frac{1}{1-\eta}},\left(\frac{4 \ell}{v} \kappa(s)\right)^{\frac{1}{1+\eta}}\right\}\right)\right]
\end{gathered}
$$

provided that

$$
T_{f} \geq 2\left(1+\frac{4}{\eta v}\right) \ell .
$$

Finally, let us formulate a useful lemma:

Lemma 1. [23] Let $x, y \in \mathbb{R}$ and $p>0$, then for any $\kappa_{1} \in(0,1)$ there exists $\kappa_{2}>0$ such that

$$
x\lceil x+y\rfloor^{p} \geq \kappa_{1}|x|^{p+1}-\kappa_{2}|y|^{p+1} .
$$

In particular, $\kappa_{2}=\max \left\{1+\kappa_{1}, \frac{\kappa_{1}}{\left(1-\kappa_{1}^{1 / p}\right)^{p}}\right\}$.

\section{Dynamic regressor extension and mixing method}

Consider the estimation problem as above:

$$
\begin{aligned}
& x(t)=\omega^{\top}(t) \theta, t \in \mathbb{R}, \\
& y(t)=x(t)+w(t) .
\end{aligned}
$$

Introduce the following hypothesis:

Assumption 1. Let $\omega \in \mathcal{L}_{\infty}\left(\mathbb{R}, \mathbb{R}^{n}\right)$ and $w \in \mathcal{L}_{\infty}(\mathbb{R}, \mathbb{R})$.

As it has been proposed in [19], in order to overcome the limitations imposed by the condition that $\omega$ is PE and also to improve the transient performance, the DREM procedure transforms (3) to $n$ new one-dimensional regression models, which allows the decoupled estimates of $\theta_{i}, i=\overline{1, n}$ to be computed under a condition on the regressor $\omega$ that differs from the persistent excitation.

For this purpose $n-1$ linear operators $H_{j}: \mathcal{L}_{\infty}(\mathbb{R}, \mathbb{R}) \rightarrow$ $\mathcal{L}_{\infty}(\mathbb{R}, \mathbb{R})$ are introduced for $j=\overline{1, n-1}$ (for instance an operator $H_{j}$ can be chosen as a stable linear time-invariant filter with the transfer function $W_{j}(s)=\frac{\alpha_{j}}{s+\beta_{j}}$, where $s \in \mathbb{C}$ is a complex variable and $\alpha_{j} \neq 0, \beta_{j}>0$ are selected to filter the noise $w$ in (3); or it can realize the delay operation with the transfer function $W_{j}(s)=e^{-\tau_{j} s}$ for $\left.\tau_{j}>0\right)$. Note that $y \in \mathcal{L}_{\infty}(\mathbb{R}, \mathbb{R})$ under Assumption 1, then these operators are applied to the measured output $y(t)$ of (3), and using the superposition principles (the operators $H_{j}$ are linear) we obtain:

$$
\tilde{y}_{j}(t)=H_{j}(y(t))=\tilde{\omega}_{j}^{\top}(t) \theta+\tilde{w}_{j}(t), j=\overline{1, n-1},
$$

where $\tilde{y}_{j}(t) \in \mathbb{R}$ is the $j^{\text {th }}$ operator output, $\tilde{\omega}_{j}: \mathbb{R} \rightarrow \mathbb{R}^{n}$ is the $j^{\text {th }}$ filtered regressor function and $\tilde{w}_{j}(t): \mathbb{R} \rightarrow \mathbb{R}$ is the new $j^{\text {th }}$ noise signal, which is composed by the transformation of the noise $w(t)$ by $H_{j}$ and other exponentially converging components related to the initial conditions of the filters. By construction $\tilde{\omega}_{j} \in$ 
$\mathcal{L}_{\infty}\left(\mathbb{R}, \mathbb{R}^{n}\right)$ and $\tilde{w}_{j} \in \mathcal{L}_{\infty}(\mathbb{R}, \mathbb{R})$ for all $j=\overline{1, n-1}$. Define new vector variables

$$
\begin{aligned}
& \tilde{Y}(t)=\left[y(t) \tilde{y}_{1}(t) \ldots \tilde{y}_{n-1}(t)\right]^{\top} \in \mathbb{R}^{n}, \\
& \tilde{W}(t)=\left[w(t) \tilde{w}_{1}(t) \ldots \tilde{w}_{n-1}(t)\right]^{\top} \in \mathbb{R}^{n}
\end{aligned}
$$

and a time-varying matrix

$$
M(t)=\left[\omega(t) \tilde{\omega}_{1}(t) \ldots \tilde{\omega}_{n-1}(t)\right]^{\top} \in \mathbb{R}^{n \times n},
$$

then stacking the original equation (3) with the $n-1$ filtered regressor models we construct an extended regressor system:

$$
\tilde{Y}(t)=M(t) \theta+\tilde{W}(t) .
$$

For any matrix $M(t) \in \mathbb{R}^{n \times n}$ the following equality is true:

$$
\operatorname{adj}(M(t)) M(t)=\operatorname{det}(M(t)) I_{n},
$$

even if $M(t)$ is singular, where adj $(M(t))$ is the adjugate matrix of $M(t)$ and $\operatorname{det}(M(t))$ is its determinant. Recall that each element of the matrix adj $(M(t))$,

$$
\operatorname{adj}(M(t))_{k, s}=(-1)^{k+s} \mathbf{M}_{k, s}(t)
$$

for all $k, s=\overline{1, n}$, where $\mathbf{M}_{k, s}(t)$ is the $(k, s)$ minor of $M(t)$, i.e., it is the determinant of the $(n-1) \times(n-1)$ matrix that results from deleting the $k^{\text {th }}$ row and the $s^{\text {th }}$ column of $M(t)$. Define

$$
\begin{gathered}
Y(t)=\operatorname{adj}(M(t)) \tilde{Y}(t), W(t)=\operatorname{adj}(M(t)) \tilde{W}(t), \\
\phi(t)=\operatorname{det}(M(t)),
\end{gathered}
$$

then multiplying from the left the extended regressor system by the adjugate matrix adj $(M(t))$ we get $n$ scalar regressor models of the form:

$$
Y_{i}(t)=\phi(t) \theta_{i}+W_{i}(t)
$$

for $i=\overline{1, n}$. Again, by construction $Y \in \mathcal{L}_{\infty}\left(\mathbb{R}, \mathbb{R}^{n}\right), W \in$ $\mathcal{L}_{\infty}\left(\mathbb{R}, \mathbb{R}^{n}\right)$ and $\phi \in \mathcal{L}_{\infty}(\mathbb{R}, \mathbb{R})$. For the scalar linear regression model (4) the conventional gradient estimation algorithm takes the form:

$$
\dot{\hat{\theta}}_{i}(t)=\gamma_{i} \phi(t)\left(Y_{i}(t)-\phi(t) \hat{\theta}_{i}(t)\right), \gamma_{i}>0
$$

for all $i=\overline{1, n}$, where now the estimation processes for all components of $\theta$ are decoupled, and the adaptation gain $\gamma_{i}$ can be adjusted separately for each element of $\theta$. However, all these estimation algorithms are dependent on the same regressor $\phi(t)$ (determinant of $M(t)$ ).

Remark 1. The choice of the operators $H_{j}$ is a degree of freedom of the DREM procedure, where a poor choice may compromise the excitation level of the original regressor $\omega$ and can yield a singular matrix $M$. In [24] it is proposed to apply the Kreisselmeier's regressor extension of the form

$$
\dot{M}(t)=-\lambda M(t)+\omega(t) \omega^{\top}(t)
$$

and

$$
\dot{\tilde{Y}}(t)=-\lambda \tilde{Y}(t)+\omega(t) y(t),
$$

where $\lambda>0$, and it is shown that under this choice the excitation of $\omega$ is always preserved.

\section{PRoblem STATEMENT}

Consider the static linear regression model (3) under Assumption 1 , and suppose that the DREM method has been applied in order reduce the initial problem of vector estimation to $n$ scalar regressor models in the form (4).

It is necessary to propose an algorithm generating an estimate $\hat{\theta}(t) \in \mathbb{R}^{n}$ of the vector of unknown parameters $\theta \in \mathbb{R}^{n}$, and for $\|W\|_{\infty}=0$ providing the property of short-fixed-time stability (see Definition 1) of the estimation error $e(t)=\theta-\hat{\theta}(t)$ dynamics under assumptions 1 for some given $T^{0}$ and $T_{f}$. If $\|W\|_{\infty} \neq 0$ then short-fixed-time ISS for $T^{0}$ and $T_{f}$ (see Definition 2) has to be guaranteed.

Since by applying DREM method the problem is decoupled on $n$ independent ones, for brevity of notation, we will further omit the index $i$ in (4) by assuming that $n=1$ :

$$
Y(t)=\phi(t) \theta+W(t)
$$

then $\theta \in \mathbb{R}, Y \in \mathcal{L}_{\infty}(\mathbb{R}, \mathbb{R}), W \in \mathcal{L}_{\infty}(\mathbb{R}, \mathbb{R})$ and $\phi \in \mathcal{L}_{\infty}(\mathbb{R}, \mathbb{R})$.

\section{DESIGN OF ESTIMATION ALGORITHMS CONVERGING IN SHORT-FIXED-TIME}

Two different solutions to the posed estimation problem are discussed in this section, whose difference consists in the requirements imposed on excitation of $\phi(t)$ and on the guaranteed robustness abilities with respect to $W(t)$.

\section{A. Algorithm 1}

Consider an adaptive estimation algorithm proposed in [25], [26]:

$$
\begin{aligned}
\dot{\hat{\theta}}(t)= & \phi(t)\left\{\gamma_{1}[Y(t)-\phi(t) \hat{\theta}(t)\rfloor^{1-\alpha}\right. \\
& \left.+\gamma_{2}\lceil Y(t)-\phi(t) \hat{\theta}(t)\rfloor^{1+\alpha}\right\}
\end{aligned}
$$

for $\gamma_{1}>0, \gamma_{2}>0$ and $\alpha \in[0,1)$, with $\hat{\theta}\left(t_{0}\right) \in \mathbb{R}$, which admits the following properties:

Proposition 1. [14] Let Assumption 1 be satisfied, and for given $T^{0}>0$ and $T_{f}>0$,

$$
\int_{t}^{t+\ell} \min \left\{|\phi(s)|^{2-\alpha},|\phi(s)|^{2+\alpha}\right\} d s \geq v>0
$$

for all $t \in\left[-T^{0}, T^{0}+T_{f}\right]$ and some $\ell \in\left(0, \frac{T_{f}}{2}\right)$. Take

$$
\min \left\{\gamma_{1}, \gamma_{2}\right\}>\frac{2^{2+\frac{\alpha}{2}}}{\alpha v\left(\frac{T_{f}}{2 \ell}-1\right)},
$$

then the estimation error $e(t)=\theta-\hat{\theta}(t)$ dynamics of (7) is shortfixed-time ISS for $T^{0}$ and $T_{f}$.

Proof. The error dynamics for the estimation algorithm (7) can be written as follows:

$$
\begin{aligned}
\dot{e}(t)= & -\phi(t)\left\{\gamma_{1}\lceil\phi(t) e(t)+W(t)\rfloor^{1-\alpha}\right. \\
& \left.+\gamma_{2}\lceil\phi(t) e(t)+W(t)\rfloor^{1+\alpha}\right\} .
\end{aligned}
$$


Consider a Lyapunov function candidate $V(e)=0.5 e^{2}$, whose derivative has an upper estimate for some $\kappa_{11}, \kappa_{12} \in(0,1)$ and $\kappa_{21}, \kappa_{22}>0$ coming from Lemma 1 :

$$
\begin{aligned}
\dot{V}(t)= & -\gamma_{1} e(t) \phi(t)\lceil\phi(t) e(t)+W(t)\rfloor^{1-\alpha} \\
& -\gamma_{2} e(t) \phi(t)\lceil\phi(t) e(t)+W(t)\rfloor^{1+\alpha} \\
\leq & -\gamma_{1} \kappa_{11}|e(t) \phi(t)|^{2-\alpha}-\gamma_{2} \kappa_{12}|e(t) \phi(t)|^{2+\alpha} \\
& +\gamma_{1} \kappa_{21}|W(t)|^{2-\alpha}+\gamma_{2} \kappa_{22}|W(t)|^{2+\alpha} \\
\leq & -u(t)\left(V^{1-\frac{\alpha}{2}}(t)+V^{1+\frac{\alpha}{2}}(t)\right)+\sigma(|W(t)|)
\end{aligned}
$$

for any $e(t) \in \mathbb{R}$ and $W(t) \in \mathbb{R}$, where

$$
\begin{gathered}
u(t)=\min \left\{2^{1-\frac{\alpha}{2}} \gamma_{1} \kappa_{11}|\phi(t)|^{2-\alpha}, 2^{1+\frac{\alpha}{2}} \gamma_{2} \kappa_{12}|\phi(t)|^{2+\alpha}\right\}, \\
\sigma(s)=\gamma_{1} \kappa_{21} s^{2-\alpha}+\gamma_{2} \kappa_{22} s^{2+\alpha}
\end{gathered}
$$

is a function from class $\mathcal{K}_{\infty}$. Note that

$$
u(t) \geq 2^{1-\frac{\alpha}{2}} \min \left\{\gamma_{1} \kappa_{11}, \gamma_{2} \kappa_{12}\right\} \min \left\{|\phi(t)|^{2-\alpha},|\phi(t)|^{2+\alpha}\right\},
$$

then under the imposed restrictions for $\phi$, the system is shortfixed-time ISS for $T^{0}$ and $T_{f}$ due to Theorem 1 provided that the constraint

$$
T_{f} \geq 2\left(1+\frac{2^{2+\frac{\alpha}{2}}}{\alpha \min \left\{\gamma_{1} \kappa_{11}, \gamma_{2} \kappa_{12}\right\} v}\right) \ell
$$

is satisfied. The imposed restriction on $\min \left\{\gamma_{1}, \gamma_{2}\right\}$ guarantees that there exist $\kappa_{11}, \kappa_{12} \in(0,1)$ such that all trajectories converge to the origin faster than $T_{f}$ if $\|W\|_{\infty}=0$.

\section{B. Algorithm 2}

And, finally, let us introduce a version of the algorithm (7), which extends the nonlinear paradigm of the former by time-varying powers:

$$
\begin{gathered}
\dot{\hat{\theta}}(t)=\operatorname{sign}(\phi(t))\left\{\gamma_{1}[Y(t)-\phi(t) \hat{\theta}(t)]^{\alpha(t)}\right. \\
+\gamma_{2}\left\lceil Y(t)-\left.\phi(t) \hat{\theta}(t)\right|^{\varsigma+\alpha(t)}\right\} \\
\gamma_{1}>0, \gamma_{2}>0, \varsigma>1, \alpha(t)=\frac{|\phi(t)|}{1+|\phi(t)|},
\end{gathered}
$$

where $\hat{\theta}\left(t_{0}\right) \in \mathbb{R}$. The idea of this design is that the power $\alpha(t)$ is approaching zero together with the regressor $\phi(t)$, then the contribution of the regressor in the adaptation rate is proportional to $|\phi(t)|^{\alpha(t)}$, which is strictly separated with zero even for a convergent regressor, the detailed proof of this fact is given below.

Proposition 2. [14] Let Assumption 1 be satisfied, and $\vartheta \in$ $\mathcal{L}_{\infty}\left(\mathbb{R}, \mathbb{R}_{+}\right)$where $\vartheta(t)=\frac{W(t)}{\phi(t)}$. Then the estimation error $e(t)=$ $\theta-\hat{\theta}(t)$ dynamics of (9) is short-finite-time ISS for any $T^{0} \geq 0$ with the input $\vartheta$.

Let additionally for given $T^{0}>0$ and $T_{f}>0$,

$$
\int_{t}^{t+\ell}|\phi(s)|^{\varsigma} d s \geq v>0
$$

for all $t \in\left[-T^{0}, T^{0}+T_{f}\right]$ and some $\ell \in\left(0, T_{f}\right)$, and

$$
\min \left\{\gamma_{1}, \gamma_{2}\right\}>\sqrt{2} \frac{1+\phi_{\max }+\frac{4 \ell}{(\varsigma-1) v}}{\left(T_{f}-\ell\right) g\left(x_{\min }\right)},
$$

where $\phi_{\max }=\max _{t \in\left[-T^{0}, T^{0}+T_{f}\right]}|\phi(t)|, g(x)=x^{\frac{x}{1+x}}$ and $x_{\min }=\operatorname{Lambert}\left(\mathbf{e}^{-1}\right)$, then the estimation error $e(t)=\theta-\hat{\theta}(t)$ dynamics of (9) is short-fixed-time ISS for $T^{0}$ and $T_{f}$ with the input $\vartheta$.
Proof. The error dynamics for the estimation algorithm (9) can be written as follows:

$$
\begin{aligned}
\dot{e}(t)= & -\operatorname{sign}(\phi(t))\left\{\gamma_{1}\lceil\phi(t) e(t)+W(t)\rfloor^{\alpha(t)}\right. \\
& \left.+\gamma_{2}\lceil\phi(t) e(t)+W(t)\rfloor^{\varsigma+\alpha(t)}\right\} .
\end{aligned}
$$

Consider a Lyapunov function $V(e)=0.5 e^{2}$ and observe that

$$
\begin{aligned}
& r^{a(t)}(t) \geq\left\{\begin{array}{ll}
r^{a_{\min }}(t) & r(t) \geq 1 \\
r^{a_{\max }}(t) & r(t)<1
\end{array} \geq \min \left\{1, r_{\min }^{a_{\max }}\right\},\right. \\
& r^{a(t)}(t) \leq\left\{\begin{array}{ll}
r^{a_{\max }}(t) & r(t) \geq 1 \\
r^{a_{\min }}(t) & r(t)<1
\end{array} \leq \max \left\{1, r_{\max }^{a_{\max }}\right\}\right.
\end{aligned}
$$

for any $r: \mathbb{R} \rightarrow \mathbb{R}_{+}$and $a: \mathbb{R} \rightarrow \mathbb{R}_{+}$such that $r_{\min }=\inf _{t \in \mathbb{R}} r(t)$, $r_{\text {max }}=\sup _{t \in \mathbb{R}} r(t), a_{\text {min }}=\inf _{t \in \mathbb{R}} a(t)$ and $a_{\max }=\sup _{t \in \mathbb{R}} a(t)$ for some $r_{\min }, r_{\max }, a_{\min }, a_{\max } \in \mathbb{R}_{+}$, then the time derivative of $V$ for the estimation error dynamics admits an upper estimate:

$$
\begin{aligned}
\dot{V}(t)= & -\operatorname{sign}(\phi(t)) e(t)\left\{\gamma_{1}\lceil\phi(t) e(t)+W(t)\rfloor^{\alpha(t)}\right. \\
& \left.+\gamma_{2}\lceil\phi(t) e(t)+W(t)\rfloor^{\varsigma+\alpha(t)}\right\} \\
= & -\gamma_{1}|\phi(t)|^{\alpha(t)} e(t)\lceil e(t)+\vartheta(t)\rfloor^{\alpha(t)} \\
- & \gamma_{2}|\phi(t)|^{\varsigma+\alpha(t)} e(t)\lceil e(t)+\vartheta(t)\rfloor^{\varsigma+\alpha(t)}
\end{aligned}
$$

$$
\begin{gathered}
\leq-\gamma_{1} \kappa_{11}|\phi(t)|^{\alpha(t)}|e(t)|^{1+\alpha(t)}-\gamma_{2} \kappa_{12}|\phi(t)|^{\varsigma+\alpha(t)}|e(t)|^{1+\varsigma+\alpha(t)} \\
+\gamma_{1} \kappa_{21}|\phi(t)|^{\alpha(t)}|\vartheta(t)|^{1+\alpha(t)}+\gamma_{2} \kappa_{22}|\phi(t)|^{\varsigma+\alpha(t)}|\vartheta(t)|^{1+\varsigma+\alpha(t)} \\
\leq-\sqrt{2} \min \left\{\gamma_{1} \kappa_{11}, \gamma_{2} \kappa_{12}\right\} g(|\phi(t)|)\left[\xi_{1}(V(t))\right. \\
\left.+|\phi(t)|^{\varsigma} \xi_{2}(V(t))\right]+\sigma\left(\|\vartheta\|_{\infty}\right),
\end{gathered}
$$

where

$$
\begin{aligned}
& \xi_{1}(s)=\left\{\begin{array}{ll}
s^{0.5} & s \geq 1 \\
s^{\frac{0.5+\phi_{\max }}{1+\phi_{\max }}} & s<1
\end{array}, \xi_{2}(s)=\left\{\begin{array}{ll}
s^{\frac{1+\varsigma}{2}} & s \geq 1 \\
s^{\frac{1+\varsigma+\frac{\phi_{\max }}{1+\phi_{\max }}}{2}} & s<1
\end{array},\right.\right. \\
& \sigma(s)=\left[\gamma_{1} \kappa_{21} \max \left\{1, \phi_{\max }^{\frac{\phi_{\max }}{1+\phi_{\max }}}\right\}\right. \\
& \left.+\gamma_{2} \kappa_{22} \max \left\{1, \phi_{\max }^{\varsigma+\frac{\phi_{\max }}{1+\phi_{\max }}}\right\} s^{\varsigma}\right] \\
& \times \begin{cases}s^{\frac{1+2 \phi_{\max }}{1+\phi_{\max }}} & s \geq 1 \\
s & s<1\end{cases}
\end{aligned}
$$

and $\kappa_{11}, \kappa_{12} \in(0,1), \kappa_{21}, \kappa_{22}>0$ are from Lemma 1 (since $\phi(t)$ is upper bounded in amplitude due to Assumption 1 such constants exist). Note that $g(0)=g(1)=1$ and

$$
\begin{gathered}
\frac{\partial g(x)}{\partial x}=\left(1+\ln (x) \frac{1}{1+x}\right) \frac{g(x)}{1+x} \\
\frac{\partial^{2} g(x)}{\partial x^{2}}=\frac{x^{2} \ln ^{2}(x)+x(x+1)^{2}}{x g(x)(x+1)^{4}}
\end{gathered}
$$

then $\frac{\partial^{2} g(x)}{\partial x^{2}} \geq 0$ for $x \geq 0$, and the function $g(x)$ has the only minimum $x_{\min } \in[0,1]$ that satisfies the equality:

$$
1+\ln \left(x_{\min }\right) \frac{1}{1+x_{\min }}=0 .
$$

Solving this equation we obtain $x_{\text {min }}=\operatorname{Lambert}\left(\mathbf{e}^{-1}\right)$, then $g\left(x_{\min }\right) \simeq 0.757$. Therefore, the estimate for the Lyapunov function can be represented as follows:

$$
\begin{aligned}
\dot{V}(t) & \leq-\varpi\left[\xi_{1}(V(t))+|\phi(t)|^{\varsigma} \xi_{2}(V(t))\right]+\sigma\left(\|\vartheta\|_{\infty}\right) \\
& \leq-\varpi \min \left\{1,|\phi(t)|^{\varsigma}\right\} \xi(V(t))+\sigma\left(\|\vartheta\|_{\infty}\right),
\end{aligned}
$$


where $\varpi=\sqrt{2} \min \left\{\gamma_{1} \kappa_{11}, \gamma_{2} \kappa_{12}\right\} g\left(x_{\min }\right)$ and

$$
\xi(s)=\left\{\begin{array}{ll}
s^{0.5}+s^{\frac{1+\varsigma}{2}} & s \geq 1 \\
s^{\frac{0.5+\phi_{\max }}{1+\phi_{\max }}}+s^{\frac{1+\varsigma+\frac{\phi_{\max }}{1+\phi_{\max }}}{2}} & s<1
\end{array} .\right.
$$

By repeating the arguments of Theorem 1, the short-fixed-time and the short-finite-time ISS [12] can be established (in the latter case there is no restriction on excitation of $\phi(t)$ and even the conventional results [27] can be used).

In order to check the restrictions on $T_{f}$ for the case $\|W\|_{\infty}=0$, assume that $V\left(t_{0}\right)>1$, denote by $T_{1}, T_{2}>0$ the instants of time such that $V\left(t_{0}+T_{1}\right)=1$ and $V\left(t_{0}+T_{1}+T_{2}\right)=0$, and use the estimate

$$
\dot{V}(t) \leq-\varpi \max \left\{V^{\frac{0.5+\phi_{\max }}{1+\phi_{\max }}}(t),|\phi(t)|^{\varsigma} V^{\frac{1+\varsigma}{2}}(t)\right\},
$$

then since $\frac{0.5+\phi_{\max }}{1+\phi_{\max }} \in(0,1)$ and $\frac{1+\varsigma}{2}>1$ we obtain

$$
V^{\frac{\varsigma-1}{2}}(t) \leq \frac{1}{V^{-\frac{\varsigma-1}{2}}\left(t_{0}\right)+\frac{\varsigma-1}{2} \frac{\varpi v}{4 \ell}\left(t-t_{0}-\ell\right)}
$$

for all $t \in\left[t_{0}, t_{0}+T_{1}\right]$, and

$$
V^{\frac{0.5}{1+\phi_{\max }}}(t) \leq 1-\frac{0.5}{1+\phi_{\max }} \varpi\left(t-t_{0}-T_{1}\right)
$$

for all $t \in\left[t_{0}+T_{1}, t_{0}+T_{1}+T_{2}\right]$. Hence, the upper bounds on $T_{1}$ and $T_{2}$ follow (due to the properties of $\alpha(t)$ the time $T_{2}$ is independent in the excitation):

$$
T_{1} \leq \ell\left[1+\frac{8}{(\varsigma-1) \varpi v}\right], T_{2} \leq 2 \frac{1+\phi_{\max }}{\varpi},
$$

which give by resolving the inequality $T_{1}+T_{2} \leq T_{f}$ the required restriction on $\gamma_{1}$ and $\gamma_{2}$.

Thus, the idea of the algorithm (9) consists in the utilization of a nonlinearity such that the function $g(|\phi(t)|)$ becomes separated with zero overcoming the absence of excitation in the system. The price for that is the robustness with respect to a noise $W$ with a well-defined ratio $\frac{W(t)}{\phi(t)}$.

Remark 2. One of the most important features of estimation algorithms, after estimation error convergence in the ideal case, is their robustness with respect to measurement noises. In our case, since the regressor $\phi(t)$ may converge to zero, the appearance of $W(t) \neq 0$ additionally limit the time of convergence, since it is reasonable to use the output $Y(t)$ for estimation with $t \in\left[t_{0}, t_{0}+T\right]$ only while

$$
|Y(t)|>|W(t)|+\varepsilon
$$

for some $\varepsilon>0$. If $Y(t)|\leq| W(t) \mid+\varepsilon$ (or $|Y(t)|$ is almost equal to $|W(t)|$ for a sufficiently small $\varepsilon$ ), then the measured output mainly contains the measurement noise, and it is ambiguous to ask an algorithm to estimate $\theta$ due to a bad ratio between the signal and the noise. In this sense, the requirement that the signal $\frac{W(t)}{\phi(t)}$ is well-defined is not much restrictive. Roughly speaking, it merely assumes that the ratio between the useful signal and the noise lies in some reasonable limits.

If the regressor $\phi(t)$ is just asymptotically converging without crossing zero, then the algorithms (7) and (9) can be applied for any finite $T>0$ and $t_{0} \in \mathbb{R}$.

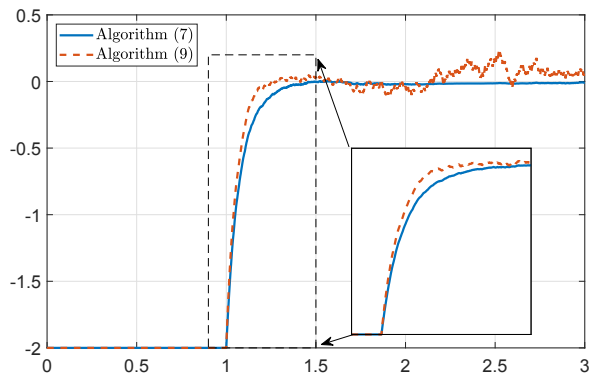

Figure 1. Simulation results for the algorithms (7) and (9), estimation error $\theta_{2}-\hat{\theta}_{2}(t)$ vs. time, seconds. The case of uniformly distributed measurement noise

\section{EXAMPLE}

Consider a measured scalar signal $y(t)$ that consists of two terms. The first term is a harmonic oscillation, and the second term is a decaying process:

$$
y(t)=\theta_{1} \sin (t)+\theta_{2} \frac{1}{t+1}+w(t),
$$

where $w(t)$ is a measurement noise, and $\theta_{1}, \theta_{2}$ are unknown constant parameters. Since the second term decays, the parameter $\theta_{1}$ can be estimated asymptotically with a standard gradient estimator; however, estimation of $\theta_{2}$ is more challenging. The signal $y(t)$ can be rewritten as (3), where the regressor $\omega(t)=\left[\sin (t) \frac{1}{t+1}\right]^{\top}$, obviously, is not persistently exciting. Thus, standard gradient or least-square approaches cannot guarantee an asymptotic estimation. This section illustrates how $\theta_{2}$ can be estimated by the means of the DREM procedure and the proposed fixed-time parameter estimation algorithms.

First, the DREM procedure is applied to get a scalar linear regression equation for $\theta_{2}$. To this end, choose the delay operator $H(u(t))=u\left(t-\tau_{d}\right)$, where $\tau_{d}>0$ is the tuning parameter, and define

$$
\tilde{\omega}_{1}(t)=H(\omega(t))=\omega\left(t-\tau_{d}\right), \quad \tilde{y}_{1}(t)=H(y(t))=y\left(t-\tau_{d}\right) .
$$

Then the procedure described in subsection II-C yields

$$
Y_{2}(t)=\phi(t) \theta_{2}+W_{2},
$$

where $Y_{2}, W_{2}$, and $\phi$ are computed as it has been explained above, and

$$
\phi(t)=\frac{\sin (t)}{1+t-\tau_{d}}-\frac{\sin \left(t-\tau_{d}\right)}{1+t}
$$

for $t \geq \tau_{d}$. Thus, the system is not persistently excited (the signal $\phi(t)$ converges to zero) and the algorithm (5) cannot be applied. However, since $\phi(t)$ decays only asymptotically, there exist $\ell$ and $v$ such that (8) and (10) are satisfied on any finite interval of time.

For simulations, the algorithms (7) and (9) are tuned as follows: $\gamma_{1}=\gamma_{2}=10, \alpha=0.25$, and $\varsigma=1.25$. All algorithms are initialized with $\hat{\theta}_{2}(0)=0$, while the true value is $\theta_{2}=-2$. For all algorithms no estimation is performed for $t \in\left[0, \tau_{d}\right], \tau_{d}=1$ since $\phi(t)$ is identically zero on this interval due to the DREM procedure.

Simulation results for the case when $w(t)$ is a uniformly distributed noise, $|w(t)| \leq 0.2$, are given in Fig. 1. As expected, all algorithms provide finite-time parameter estimation. The algorithm (7) has both small mean error and small error oscillations, where the trade-off is the largest transient time for similar tuning parameters. 


\section{CONCLUSIONS}

The problem of estimation in the linear regression model has been considered on a bounded interval of time. Two fixed-time converging estimators are presented. In order to analyze the robustness of these estimation algorithms, a short-time fixed-time input-to-state stability property has been used. The obtained estimation algorithms are compared in numerical experiments.

\section{REFERENCES}

[1] X. Yan, Linear Regression Analysis: Theory and Computing. Singapore: World Scientific, 2009.

[2] A. Rencher and W. Christensen, Methods of Multivariate Analysis, 3rd ed., ser. Wiley Series in Probability and Statistics. New Jersey: John Wiley \& Sons, 2012, vol. 709.

[3] S. Sastry and M. Bodson, Adaptive Control: Stability, Convergence and Robustness. London: Prentice-Hall, 1989.

[4] H. K. Khalil, Nonlinear Systems, 3rd ed. Upper Saddle River, New Jersey: Prentice Hall, 2002.

[5] K. Narendra and A. Annaswamy, "Persistent excitation in adaptive systems," International Journal of Control, vol. 45, no. 1, pp. 127160, 1987.

[6] D. Efimov and A. Fradkov, "Design of impulsive adaptive observers for improvement of persistency of excitation," Int. J. Adaptive Control and Signal Processing, vol. 29, no. 6, pp. 765-782, 2015.

[7] L. Praly, "Convergence of the gradient algorithm for linear regression models in the continuous and discrete time cases," December 26 2016, int. Rep. MINES ParisTech, Centre Automatique et Systèmes. [Online]. Available: https://hal.archives-ouvertes.fr/hal-01423048

[8] N. Barabanov and R. Ortega, "On global asymptotic stability of $\dot{x}=-\phi(t) \phi^{\top}(t) x$ with $\phi(t)$ bounded and not persistently exciting," Systems \& Control Letters, vol. 109, pp. 24-27, 2017.

[9] D. Efimov, N. Barabanov, and R. Ortega, "Robust stability under relaxed persistent excitation conditions," International Journal of Adaptive Control and Signal Processing, 2019.

[10] G. Chowdhary, T. Yucelen, M. Mühlegg, and E. N. Johnson, "Concurrent learning adaptive control of linear systems with exponentially convergent bounds," International Journal of Adaptive Control and Signal Processing, vol. 27, no. 4, pp. 280-301, 2012.

[11] R. Kamalapurkar, P. Walters, and W. E. Dixon, "Model-based reinforcement learning for approximate optimal regulation," Automatica, vol. 64, pp. 94-104, 2016.

[12] J. Wang, D. Efimov, and A. Bobtsov, "On robust parameter estimation in finite-time without persistence of excitation," IEEE Transactions on Automatic Control, vol. 65, no. 4, 2020.

[13] A. Belov, S. Aranovskiy, R. Ortega, N. Barabanov, and A. Bobtsov, "Enhanced parameter convergence for linear systems identification: The DREM approach," in Proc. European Control Conference (ECC), Limassol, 2018.

[14] J. Wang, D. Efimov, S. Aranovskiy, and A. Bobtsov, "Fixed-time estimation of parameters for non-persistent excitation," European Journal of Control, 2019.

[15] V. Andrieu, L. Praly, and A. Astolfi, "Homogeneous Approximation, Recursive Observer Design, and Output Feedback," SIAM J. Control Optimization, vol. 47, no. 4, pp. 1814-1850, 2008.

[16] E. Cruz-Zavala, J. Moreno, and L. Fridman, "Uniform robust exact differentiator," IEEE Transactions on Automatic Control, vol. 56, no. 11, pp. 2727-2733, 2011.

[17] A. Polyakov, "Nonlinear feedback design for fixed-time stabilization of linear control systems," IEEE Transactions on Automatic Control, vol. 57, no. 8, pp. 2106-2110, 2012.

[18] Y. Song, Y. Wang, J. Holloway, and M. Krstic, "Time-varying feedback for regulation of normal-form nonlinear systems in prescribed finite time," Automatica, vol. 83, pp. 243-251, 2017.

[19] S. Aranovskiy, A. Bobtsov, R. Ortega, and A. Pyrkin, "Performance enhancement of parameter estimators via dynamic regressor extension and mixing," IEEE Transactions on Automatic Control, vol. 62, no. 7, pp. 3546-3550, 2017.

[20] H. Ríos, D. Efimov, A. Polyakov, and W. Perruquetti, "Homogeneous time-varying systems: Robustness analysis," IEEE Transactions on Automatic Control, vol. 61, no. 12, pp. 4075-4080, 2016.
[21] Y. Hong, " $\mathrm{H}_{\infty}$ control, stabilization, and input-output stability of nonlinear systems with homogeneous properties," Automatica, vol. 37, no. 7, pp. 819-829, 2001.

[22] E. Bernuau, A. Polyakov, D. Efimov, and W. Perruquetti, "Verification of ISS, iISS and IOSS properties applying weighted homogeneity," Systems \& Control Letters, vol. 62, pp. 1159-1167, 2013.

[23] J. G. Rueda-Escobedo, D. Ushirobira, R. Efimov, and J. A. Moreno, "Gramian-based uniform convergent observer for stable LTV systems with delayed measurements," Int. J. Control, 2019. [Online]. Available: https://hal.inria.fr/hal-01889193

[24] M. Korotina, S. Aranovskiy, R. Ushirobira, and A. Vedyakov, "On parameter tuning and convergence properties of the DREM procedure," in Proc. European Control Conference, Saint-Petersburg, 2020, submitted.

[25] H. Ríos, D. Efimov, J. A. Moreno, W. Perruquetti, and J. G. RuedaEscobedo, "Time-varying parameter identification algorithms: Finite and fixed-time convergence," IEEE Transactions on Automatic Control, vol. 62, no. 7, pp. 3671-3678, 2017.

[26] H. Ríos, D. Efimov, and W. Perruquetti, "An adaptive sliding-mode observer for a class of uncertain nonlinear systems," International Journal of Adaptive Control and Signal Processing, vol. 32, no. 3, pp. 511-527, 2018.

[27] E. Sontag and Y. Wang, "On characterizations of the input-to-state stability property," Systems \& Control Letters, vol. 24, pp. 351-359, 1995. 